Compte tenu de l'évolution démographique, la question de l'addiction chez les personnes âgées sera de plus plus présente à l'avenir dans les débats autour de l'efficacité de la prise en charge. D'une part, la complexité des maladies de la dépendance chez les personnes âgées nous posera d'importants défis face à la détérioration de la prise en charge qui s'annonce dès 2018 dans les soins ambulatoires au cabinet et en institution et à la limitation massive de la collaboration interprofessionnelle suite à l'intervention tarifaire prévue par le Conseil fédéral, et face à la pénurie de personnel spécialisé. D'autre part, les stratégies et mesures de prévention spécifiques à cette catégorie de la population doivent être développées et implémentées rapidement afin d'avoir un impact aussi positif que possible sur la qualité de vie des personnes concernées et de soulager les proches et les soignants. Les ressources nécessaires et les coûts continueront d'augmenter en raison du manque de responsabilité du Parlement dans le domaine de la prévention structurelle. Avec l'écart qui se creuse entre besoins accrus et rationalisation prévisible des prestations médicales pour les personnes vulnérables, la participation au congrès dédié à cette question en vaut donc la peine.

\title{
Vieillissement et addiction: bien soigner quand même?
}

\section{Gerhard A. Wiesbeck}

Prof. Dr méd., Société Suisse de Médecine de l'Addiction (SSMA), Membre du comité d'organisation de la conférence «Vieillissement et addiction: bien soigner quand même?", Membre FMH

\section{Références}

1 Bitar R, Dürsteler KM Rösner S, Grosshans M, Herdener M, et Mutschler J. Substance abuse in older adults. Praxis 2014:103(18):1071-9.

Notari L, Le Mével L, Delgrande Jordan M, et Maffli E. Zusammen fassende Ergebnisse der Schweizerischen Gesundheitsbefragungen 2012, 2007, 2002, 1997 und 1992 hinsichtlich des Konsums von Tabak, Alkohol, Medikamenten und illegalen Drogen. Addiction Suisse. 2014: Forschungsbericht $\mathrm{N}^{\circ} 70$.

NIDA. Substance Abuse Among Older Adults. https://www.drugabuse gov/news-events/nidanotes/2011/12/substanceabuse-among-older-adults (Publication en ligne). 2011, 19 décembre. Dernier accès: $1^{\text {er juin }} 2017$.

4 Wetterling T, et Junghanns, K. (2017). Substance abuse in older psychiatric inpatients. SUCHT. 2017;63(2):115-21.

\section{Correspondance:}

Prof. Dr méd.

Gerhard A. Wiesbeck

Société Suisse de Médecine

de l'Addiction (SSMA),

Membre du comité d'organi-

sation de la conférence

"Vieillissement et addiction: bien soigner quand même?» Universitäre Psychiatrische Kliniken Basel

Wilhelm Klein-Strasse 27

CH-4012 Bâle
Il n'y a pas d'âge pour être dépendant. Dans la mesure où nous sommes de plus en plus nombreux à vivre de plus en plus longtemps, le nombre de personnes âgées en situation d'addiction est appelé à augmenter au cours des prochaines années. Cela représente un défi à relever pour toutes les personnes concernées ou impliquées. L'acool et les médicaments occupent la première place parmi les substances psychotropes les plus consommées par les personnes âgées. Selon les observations, les hommes âgés ont davantage tendance à connaître des problèmes d'alcool, les femmes âgées des problèmes liés aux médicaments [1]. Chez les deux sexes, bien que les intoxications alcooliques diminuent de manière continue, la consommation à risque continue d'augmenter. En clair, les personnes âgées consomment de l'alcool de manière moins excessive, mais fréquente. Il n'est pas rare qu'ils consomment à la fois de l'alcool et des médicaments [2].

De nombreux facteurs aggravants liés à lâge viennent favoriser l'émergence d'une dépendance au cours du dernier tiers de la vie. Parmi ceux-ci, citons la fin de la vie professionnelle, le décès des amis ou de son/sa conjoint-e, la diminution des capacités physiques et mentales, et une confrontation croissante avec la maladie et la mort [3]. Les addictions chez les personnes âgées sont difficiles à identifier de manière précoce. Elles se cachent souvent derrière des symptômes considérés comme «typiques du vieillissement»: chutes, confusion, troubles de l'attention et de la concentration, négligence de l'hygiène corporelle, troubles de l'alimentation et symptômes dépressifs, autant de manifestations dont on peut facilement concevoir la multiplication avec l'âge. En Suisse, les quelque 20000 personnes dépendantes aux opioïdes de substitution, toujours plus nombreux à se rapprocher du troisième âge, constituent un défi particulier. Les maladies typiquement liées à l'âge interviennent plus tôt et plus fréquemment chez eux que dans le reste de la population. Ils connaissent un vieillissement biologique précoce marqué, souffrent de nombreuses comorbidités physiques et psychiques, et font peser une forte demande sur le système de santé et d'accompagnement du vieillissement [4].

De nombreuses professions s'occupent des personnes âgées en situation d'addiction: des domaines aussi divers que la politique, le travail social, la psychologie, la médecine, les soins aux malades et aux personnes âgées, le conseil nutritionnel etc. sont impliqués. Ce sont autant de représentations et de concepts divers à rapprocher, prioriser et coordonner; au cours de ce processus, les souhaits des premiers concernés et de leurs proches peuvent souvent passer au second plan. Du fait de cette interdisciplinarité, et de la multiplicité des intérêts qui en découlent, il est urgent et nécessaire de créer des modèles efficaces de soin pour les personnes âgées souffrant d'une addiction.

C'est à cette nécessité qu'est consacrée la conférence «Vieillissement et addiction: bien soigner quand même?» organisée par Fachverband Sucht en coopération avec de nombreuses associations professionnelles le 30 août 2017. Pour la première fois, les représentants des personnes concernées et des acteurs impliqués se rencontreront autour du thème de l'addiction et du vieillissement. La conférence abordera notamment les possibilités, les avantages et les limites des structures de soin, d'accompagnement des addictions et du vieillissement. Des modèles adaptés à la conjoncture actuelle seront présentés, les mesures indispensables soulignées. Cette conférence vise à promouvoir l'échange et le dialogue interdisciplinaires. 